Stor Fjord, the expedition made for Ice Fjord, which, at its east end, sends off three branches. A careful examination was made of several parts of the coast of this fjord, and in the neighbourhood of the North Fjord Nordenskjöld collected a large number of triassic fossils, among them large nautilus-like shells and fragments of bones, some of which were thought to have been four feet long, belonging to crocodile-like animals. While in Ice Fjord, the expedition met with Mr. Birkbeck's yacht Sultana, " a beautiful but fragile nutshell," having among others on board, Prof. Newton. Here also a water-mark was fixed. After leaving Ice Fjord, the Axel Thordsen succeeded in reaching Stans Foreland, a large island to the south-east of the main island, having on its south the Thousand Islands.

"Fortunately it appeared that the discouraging descriptions of the fogs prevailing here are properly applicable to the Thousand Islands, comparatively clear weather, on the contrary, being common in the inner part of the fjord. Here, as at many other places on Spitzbergen, may be found cloudless skies and sunshine, while an impenetrable fog lies at the mouth. The cause of this is to be sought for in the course of the marine currents. While an arm of the Gulf Stream, as the masses of drift-wood heaped up at South Cape and the Thousand Islands show, at least during a portion of the year, flows past the southern part of West Spitzbergen and Stans Foreland, it is the Arctic current entering from Helis Sound and Walter Thymen's Strait, which principally prevails in the interior of Stor Fjord. There is, therefore, no drift-wood to be met with on the shores of this fjord, on which account it is necessary on boat voyages to carry a supply of fuel. During boat voyages along the north coast of Spitzbergen one may, on the contrary, nearly always reckon on finding in the neighbourhood of the restingplace dry and excellent material even for a large log fire."

The west coast of Stor Fjord is occupied by enormous glaciers, which go down to the sea and are only interrupted by black, often conically-shaped mountain tops. On the east coast, on the contrary, between Whales' Point and Helis Sound, there is only a single considerable glacier, the coast being formed of a continuous rocky wall, which rises almost directly from the sea to a snowfree plateau of about 1,000 feet in height. At the foot of this wall there are here and there grassy slopes, which form the finest reindeer grounds on Spitzbergen. The west coast both of Stans Foreland and of Barents Land was examined as far north as the extremity of the fjord. In connection with this part of the expedition's work, some interesting remarks are made on the character of the Spitzbergen glaciers:-

"When icebergs are spoken of in the region of Spitzbergen, it ought to be remembered that what is meant is large blocks of ice which fall down from the perpendicular sea faces of the glaciers. Though these blocks are often exceedingly large, they cannot in any way be compared with the icebergs in the Greenland waters, which are said to reach a height of 1,000 feet. The glaciers on Greenland near the sea are indeed higher than on Spitzbergen, but this dissimilarity is not sufficiently great to explain the great difference in the dimensions of the glaciers at the two places. There is great probability in Prof. Edlund's hypothesis that the larger icebergs are formed by blocks of ice falling down from a glacier coming in contact in their lower parts with an over-cooled stratum of water which, as is well known, in contact with actual ice immediately assumes the solid form. For any such over-cooled stratum of water can, on account of the Gulf Stream, only exceptionally occur on the coasts of Spitzbergen, while the contrary is the case in the waters of Greenland, which are taken up almost exclusively by the Arctic current. The ice seeds which have fallen from the glaciers thus find only at Greenland a suitable soil for their further development, only there do they grow to enormous ice-masses, which are so often the cause of the navigator's astonishment and alarm."

(To be continued)

\section{HERING'S THEORY OF THE VISION OF LIGHT AND COLOURS}

A FEW years ago Herr Ewald Hering, Professor of -1 Physiology at Prague, communicated to the Imperial Academy of Sciences at Vienna a series of six papers propounding a new explanation of the physiology of vision, so far as concerns the perception of light and colour. The papers were subsequently collected and published in a separate form ${ }^{1}$ and have had a wide circulation. The author is well known by his researches on various "physiological subjects, and has long devoted attention specially to the phenomena of vision, many of his views having been discussed at much length in Helmholtz's "Handbook of Physiological Optics," published between 1856 , and I 866 .

The principles developed in the papers in question have attracted much attention on the Continent, and, it is believed, have been thought well of by many competent authorities. So far as I know, however, no account of them, beyond meagre notices of a few lines, has yet been made accessible to English readers. It is highly desirable, both for the reputation of the author and for the information of those of our countrymen who are interested in the subject, that this want should be supplied, and I propose now to offer to the readers of NATURE an abstract of Prof. Hering's theory, sufficiently explicit to enable its general nature to be understood, but at the same time not so full as to supersede reference to the work itself by those who desire to appreciate the reasoning more thoroughly.

The theory of the perception of light and colours at present best known and most generally adopted, is the one formed on the views of Thomas Young, and further elaborated by Helmboltz in his great optical work-hence called the Young-Helmholtz theory. That which Hering proposes to substitute for it may rather be considered as an extension and an improvement than an opposing theory, inasmuch as its chief aim is, by the introduction of additional elements, to account for phenomena which, according to the previous hypotheses, are left obscure, or receive insufficient explanations. This consideration will insure for the theory a more favourable reception than if its object were completely to overturn received ideas. It is not, however, intended here to offer any discussion of the theory; we have only to state what its general features are.

In the first place, it should be explained that the theory is developed chiefly in regard to the vision of black and white, and their mixture, gray. The subject of colour is introduced afterwards, following out the same principles.

The reasoning is founded for the most part on a class of visual phenomena of a subjective nature, such as the effect of contrasts, appearances after looking steadfastly at objects, and so on. These phenomena have long been considered important in regard to the theory of vision, and they are treated of by Helmholtz at much length. The author, however, contends that the endeavours made to explain them have been hitherto imperfect, inasmuch as it has been necessary to call in for this purpose the aid of psychological considerations, such as the effect of imagination and other causes of deception. As he expresses it, on every other page of a professed treatise on physiological optics, one finds the mental judgment invoked as a deus ex machinat to explain any sort of diff-

I "Zur Lehre vom Lichtsinne.". Sechs Mittheilungen an die kaiserl. A kademie der Wissenschaften in Wien. Von Ewald Hering, Professor der Physiologie in Prag. Zweiter Abdruck. Wien, 1878 . 
culty. He objects to this, alleging that these phenomena ought to be capable of purely physiological explanations, ought to be capable of purem properly investigated, they afford the best means of determining the true character of light-vision.

Acting on this idea, he devotes his first three papers to an examination of these subjective phenomena. He an examination of experiments of great simplicity, illustrating the various points of importance, and he founds trating the as he goes on, a chain of reasoning which upon them, as entually to the statement of his doctrine. It will be necessary briefly to notice this introductory matter.

The first paper treats of what the author calls "Successive Light-Induction," which he illustrates in several different ways.

If you look steadfastly for, say, fifteen seconds to a minute, at the centre of a small white circular disk, laid on a large black ground (white paper on dull black velvet is best), then, closing and covering the eyes, you will soon perceive a negative after-image ( $N$ achbild $)$ of what you have seen. The disk itself will appear dark, generally much darker than the general visual area, and will be sharply defined, but it will be surrounded by a peculiar "light-space" (Lichthof), brightest close to the disk, and becoming gradually darker as it recedes, until it fades into the general dark area around. The appearance is that of a halo, or more exactly that of the sun during a total eclipse, where the intensely black circle is seen surrounded by the corona.

This phenomenon is usually explained as follows:There is a certain internal light-stimulus which acts when the eyes are closed; but the part of the retina which has been exposed to the bright light from the disk has become fatigued thereby and is less sensitive to the internal lightstimulus than the parts around; so that the fatigued circle appears darker than elsewhere. This explanation, which is purely physiological, answers very well so far as the disk is concerned, but it gives no account of the surrounding light-space, for which the psychical deus ex machina $\hat{a}$ is called in, it being asserted that the halo is only a mental delusion caused by the contrast between the dark circle and the less dark space around. The author objects altogether to the sufficiency of this suggestion, and has devised an experiment to show that the appearance of the light-space is a real and not an imaginary one.

He proves that when the light-spaces of two neighbouring dark negative after-images are superposed, they cause an increase of brightness. To show this, cut out two squares of white paper and place them side by side on the black ground, leaving a small interval between them. Observe then, as before, fixing the eyes steadfastly on a point in the black interval. In the after-image it will be noticed that the narrow intervening strip is much brighter than the space around the other three sides of each square, showing the effect of the superposition in that place of the two light-spaces together.

For a further study, examine the negative after-image of a narrow strip of black paper laid on a white ground; and direct attention first to the absence of any partial darkening of the general ground close to the strip (which ought to result if it were merely the impression of contrast), and, secondly, to the intensity of the brightness of the image of the strip itself. Lastly, to prove that this brightness is far greater than can be due to the self-working inner light-excitation of the retina, an ingenious experiment is shown (easy to carry out, but somewhat lengthy to describe), by which an objective test of the strength of the illumination can be made. The negative light after-image of a black stripe is found, by direct comparison, to be much brighter than a certain amount of objective light thrown on a fresh and unused part of the field of vision, proving, therefore, that the former must be due to something more than the mere unfatigued inner light of the retina.

In concluding this paper the author gives some general conclusions from these experiments. From an analogy with certain effects of colour, he names the light produced in the after-image, around a dark area, induced light, and the general effect successive light-induction. This takes place on any part of the retina where there has, in the object originally looked at, been a boundary between light and dark, the light part inducing an impression of light in the neighbouring part, so that when the eyes are shut the latter appears bright. The induced light is naturally most powerful in the immediate neighbourhood of the boundary, and diminishes as the distance from this increases, until it fades into the general aspect of the visual field. The author draws two important conclusions from this part of the investigation: (I) that the activity of one part of the retina corresponds with that of others, the usual idea of the independence of each part being untrue; (2) that the so-called independent or inner light of the retina is capable, within certain physiological limits, of being considerably increased in intensity. The phenomena of light-induction are subsequently examined more fully from a more general point of view; in the meantime it is considered as sufficiently shown that the ordinary fatigue theory is not sufficient to explain the appearances observed.

The second memoir is on "Simultaneous Light-Contrast." The author begins with the simplest example :-If a strip of gray paper is placed alternately against a black and a white background, in the former case it will appear much lighter than in the latter. The psychical theory explains this as a false mental judgment, assuming that the absolute sensation conveyed by the gray paper to the eye is the same in both cases. The author controverts this, insisting that the impression is, as a matter of reality and not of imagination, different in the two cases, which he proves by the following experiment :-Make a ground half white and half black, and lay two narrow strips of the same gray paper"parallel to each other and a short distance apart, one on each half of the ground. The one on the white ground will look so much darker than the other, that a new-comer would scarcely believe they were both the same shade. Now fix the eyes for half a minute or a minute intently on a point midway between the strips; then close and darken the eyes and observe the afterimage. The difference in luminosity of the two strips will be even greater than the apparent difference of shade in the original. And as this after-image cannot recognise any matter of judgment, but only faithfully records the actual state of the retina, it proves that the difference in shade observed was real, and not imaginary; and the inapplicability of the false-judgment-by-contrast explanation is manifest by the fact that the difference between the two strips will often remain after the background, forming the contrast, has disappeared.

The author then goes on to give his explanation of simultaneous contrast, namely, that the light-perception of any part of the retina depends not only on the illumination of that part, but also on the illumination of other parts adjoining. He adds that as in modern physiology it is customary to explain the varying strength of reaction consequent on a uniform stimulus, as varying excitability (Erregbarkeit), it may be said that here the effect of contrast is due to the fact that the excitability, and consequently the excitement, of one part of the retina is a function of the contemporary illumination of the whole retina, or at least of the parts immediately around.

The next step is to point out the connection that exists between the effect of contrast treated of here, and the light-induction described under the former head. The phenomena of successive light-induction showed that a part 
of the retina illuminated either not at all or very faintly, but whose immediate neighbourhood was brightly illuminated, gave, in the negative after-image, a much stronger brightness than one, the neighbourhood of which was not so illuminated. The phenomena of contrast show, on the other hand, that the light-perception of a weakly illuminated part of the retina is diminished when its surrounding part is brightly illuminated. Thus the lowering of the perception of brightness (or excitement) during the observation of an object, corresponds to the raising of this perception in the after-image, so that the successive light-induction appears as the opposite or reversal (Gegensatz) of the simultaneous contrast-action. And the latter may be denoted as a simultaneous negative light-induction.

It is, therefore, easy to imagine an internal causal connection between both phenomena, and to consider the lowering of the light-perception (excitability) which takes place during the contrast, as the cause of the raising of the perception which takes place afterwards.

The third memoir treats of "Simultaneous Light-Induction" and "Successive Contrast." To illustrate the former, look fixedly at any point in a line dividing a black space from a white one; after looking some time you will see that the originally deep black begins to lighten into gray; the brightening is the greatest close to the division line, and diminishes farther away, but after sufficiently long observation it will extend more or less over the whole black field. A contrary effect takes place, but less prominently, in the other half, the white darkening in a similar way.

This appearance is usually explained in the following half physiological, half psychological way. From the increasing fatigue of the retina, the white sensation becomes gradually weaker, and, as a consequence of this, the contrast action between the white and the black becomes weaker, and the latter appears lighter, through the same false judgment invoked to explain contrasts generally.

To show the insufficiency of this explanation the following experiment is proposed. Put a strip of black velvet on a white ground, well lighted by a gas flame; after observing it steadfastly for a time gradually diminish the light, and it will be seen that the black strip becomes brighter, and the white ground darker, until at length the former appears actually lighter than the latter. If the explanation is applied to this it must be assumed that the fatigue of the retina has become so great that the moderated white produces actually a less powerful impression than the black stripe, which gives out light of very small intensity, or in other words, that the power of the retina is so crippled that a moderate light is no longer able to produce an impression equal to the inner selfillumination of the unfatigued organ. This can be also shown by another trial which is described.

A further experiment enables a direct comparison to be made. In the middle of a broad white stripe laid on a black ground, cut a longitudinal opening; this will show an internal black stripe, surrounded by a white surface which is again surrounded by a black ground: then fix the eyes on the middle of the figure, and, after observing some time, gradually reduce the light. It will soon be found that the black internal stripe appears lighter than the external black ground, although both are objectively equally dark, and the parts of the retina brought into action are, as it is said, equally free from fatigue.

The changes consequent on the reduction of the illumination show that the simultaneous light-induction passes over into the successive, the latter being, indeed, only a more clearly manifested continuation of the former. The connection of the three phenomena hitherto treated of may be illustrated simply as follows :-When you first observe a line of division between dark and light, the dark part, especially near the division, appears darker than it would do if the white were absent; this is simultaneous contrast. Continue the fixed observation a longer time, and the darkening gradually changes to a contrary effect of brightening, which also is most powerful near the division; this is simultaneous light-induction. Lastly, close and darken the eyes, and this brightened space continues a long time visible in the after-image. This is successive light-induction.

The last of the subjective phenomena treated of by the author he calls "Successive Light Contrast." It differs from the three last mentioned in that, while they all refer to the comparison of one visual space with another close adjoining, this refers to a comparison of the impressions derived from the same space at two successive times. The simplest example is as follows:-Put a strip of white paper on a black ground, and observe it steadfastly for a time ; then let it be suddenly removed, keeping the eyes fixed on the same spot. A negative after-image will result, of a changeable character, but showing generally a space corresponding to the paper, much darker than the already dark ground. This is very striking; an inexperienced observer will be loth to believe that he can see anything darker than the blackest velvet; but there can be no mistake about the impression conveyed. The usual explanation of this is that the part of the retina stimulated by the white paper is more fatigued than the rest, and therefore, after it is removed, is less sensitive to the faint light given off by the black ground, making it thus appear still fainter. The author deems this explanation insufficient, as it is inconsistent with many of the changing phases observed in the after-image; and he describes several other experiments to support this view. He points out that the "successive light-induction," before described, may play an important part in these phenomena, but he does not attempt here to give any complete explanation of them.

It is pointed out at the close of this part of the subject that many of the experiments may be made with colours, instead of with simple light and shade, it being understood that black and white hold the same relation to each other, for this purpose, as blue and yellow, or red and green.

(To be continued.)

WILLIAM POLE.

\section{ALFRED HENRY GARROD}

$\mathrm{I}^{\mathrm{T}}$ has seldom been our lot to have to record the premature close of a career so full of promise as that which ended with the death of Alfred Henry Garrod, at his father's house in Harley Street, on Friday last, October I7. The son of an eminent physician, Dr. Alfred Baring Garrod, F.R.S., he was born in London on May I8, 1846, received a medical education at King's College, London, and in 1868 entered St. John's College, Cambridge. He graduated (B.A.) in $187 \mathrm{r}$, taking the highest place in the natural science tripos. In due course he took his M.A. degree, and was elected a Fellow of his college in 1873 His earliest scientific predilections were chiefly for mathematics and physics, and the knowledge of these subjects which he acquired when a student was of great value to him in his biological researches. The mechanics of physiology was the subject to which he first turned his attention as a scientific investigator, and, while still an undergraduate, he communicated a paper on the cause of the diastole of the ventricles of the heart to the Fournal of Anatomy (vol. iii., 1869). About the same time he sent to the Royal Society the results of an interesting series of experiments made upon himself with a view of ascertaining the causes of the minor fluctuations in the temperature of the human body while at rest, from which he concluded that these fluctuations mainly result from alterations in the amount of blood exposed at the surface to the influence of absorbing and conducting 